\title{
Validation of New Potential Targets for Remission and Low Disease Activity in Psoriatic Arthritis in Patients Treated with Golimumab
}

Laura C Coates MD ${ }^{1}$, Proton Rahman $\mathrm{MD}^{2}$, Eliofotisti Psaradellis $\mathrm{PhD}^{3}$, Emmanouil Rampakakis $\mathrm{PhD}^{3}$, Brendan Osborne $\mathrm{PhD}^{4}$, Allen Lehman $\mathrm{PhD}^{4}$, Francois Nantel $\mathrm{PhD}^{4}$

1: Nuffield Department of Orthopaedics, Rheumatology and Musculoskeletal Sciences, University of Oxford, Oxford, United Kingdom. 2: Memorial University, Faculty of Medicine, St Johns, NL, Canada. 3: JSS Medical Research, Montreal, QC, Canada. 4: Medical Affairs, Janssen Inc., Toronto, ON, Canada

Author for correspondence: Francois Nantel, Janssen Inc. 19 Green Belt drive, Toronto, ON, M1C 1L9, Canada. fnantel@its.jnj.com

Running title: New targets for psoriatic arthritis

This study was financed by Janssen Inc. 
New targets for psoriatic arthritis

\begin{abstract}
Objective: Treat to target recommendations for psoriatic arthritis (PsA) state that the target of treatment should be remission or, at the very least, low disease activity (LDA). Different clinical indexes have been proposed to define these disease states including the minimal disease activity (MDA) criteria and the Disease Activity in PsA (DAPSA) scores which have 7 and 4-5 domains, respectively.

Using a Canadian cohort, the objectives were to calculate the proportion of patients achieving these criteria, their prognostic value and the overall patient impact of these disease states.

Methods: BioTRAC is an ongoing, prospective registry of inflammatory arthritis patients. A total of 188 patients PsA patients treated with golimumab were included. Data collected at baseline, 6 and 12 months were used.

Results: Between 15.6-38.3\% of patients achieved remission, and 37.4-77.7\% achieved LDA at 6 and 12 months' follow-up. Patients achieving any MDA target and DAPSA LDA had significantly lower SJC, TJC, PASI, dactylitis and enthesitis scores compared to non-achievers $(\mathrm{p}<0.05)$. Higher HAQ scores $(\mathrm{p}<0.03)$ were observed in patients achieving remission with remaining dactylitis or active skin disease.
\end{abstract}

Conclusion: Very Low Disease Activity (VLDA) was the most stringent new potential target for remission in PsA. There was a high level of agreement between scores although residual activity in dactylitis and skin despite DAPSA remission may impact patient function. Patients achieving either DAPSA endpoint, however, did not show a significant reduction in skin disease indicating that those two criteria are more restricted to joint symptoms. 
New targets for psoriatic arthritis

\section{INTRODUCTION}

Treat to target recommendations for psoriatic arthritis (PsA) state that the target of treatment should ideally be remission or alternatively inactive disease [1,2]. At the time of publication, the definition of remission was a complete absence of disease activity with no signs or symptoms of in all domains. However, there was no agreed definition for low disease activity which could be used as an alternative target if remission is unfeasible [3]. Several composite scores for PsA have been developed over the years and there remains considerable debate as to which should be used to define those appropriate disease states [4-7]. The Minimal Disease Activity (MDA) criteria include multiple domains such as TJC and SJC, skin, enthesitis, patient pain and global disease activity scores and the Health Assessment Questionnaire (HAQ) [8]. Conversely, the Disease Activity Index for Psoriatic Arthritis (DAPSA) focuses on peripheral arthritis and includes tender and swollen joints (TJC, SJC), C-reactive protein (CRP) and patient-reported pain and global disease activity scoring. The clinical DAPSA (cDAPSA) was adjusted later to exclude the CRP [9].

Both measures have been used to define remission or low disease states. For MDA, a modified version, the very low disease activity (VLDA), was developed to be used as a remission target [3]. Other modified versions of the MDA, with a focus on joint and skin symptoms, were developed to be used as alternative low disease activity targets [7, 10]. Specific cut-off values to define remission or low disease activity were also developed for DAPSA and cDAPSA [11].

There is only scarce data comparing these measures [7] and it is unknown if they reflect similar clinical disease activity on the various disease domains along with their impact on function and long term outcomes.

Using an existing real world cohort of Canadian PsA patients allowing calculation of remission and LDA, the objectives of this study were to evaluate the proportion of patients achieving these criteria, their prognostic value and the overall impact of these disease states on patient outcomes. 
New targets for psoriatic arthritis

\section{METHODS}

\section{Study Design}

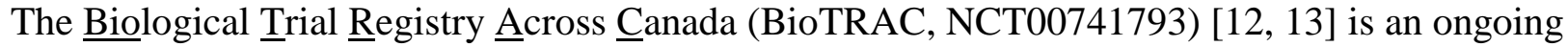
prospective, multicentre, observational registry collecting real-world clinical and safety data in patients with rheumatoid arthritis, axial spondyloarthritis and PsA treated with infliximab, golimumab (GLM) or ustekinumab as part of their routine care. In accordance with the observational nature of the registry, there is no protocol-defined intervention in the patient management and all clinical decisions and treatments are based on routine practice and the judgement of the treating physicians. Patients provided written informed consent prior to participation in the study. Ethics approvals for participation in the BioTRAC program were obtained from the respective Research Ethics Boards of participating institutional sites and a Central Institutional Review Board (IRB Services, Ontario Canada) for private practice sites. BioTRAC is conducted per the tenets of the Declaration of Helsinki was initiated in February 2002 and is ongoing.

For this study, we included 201 PsA patients treated with GLM from 2010 to 2017. The following clinical/laboratory parameters and patient reported outcomes were collected at baseline and at every 6 months: morning (AM) stiffness, swollen joint count (SJC28), tender joint count (TJC28), patient's global assessment (PtGA) and physician's global assessment of disease activity (MDGA), Health Assessment Questionnaire (HAQ), patient's assessment of pain, C reactive protein (CRP), erythrocyte sedimentation rate (ESR), dactylitis, enthesitis (16 locations including supraspinatus, medial and lateral epicondyle humerus, greater trochanter, quadriceps to patella, patella to tibia, Achilles and plantar fascia) and Psoriasis Area and Severity Index (PASI).

\section{Statistical Methods}

The proportions of patients in remission and LDA was descriptively assessed at baseline, 6 and 12 months. Three potential definitions of remission were used:

1. VLDA where all seven of the MDA cut points were required to be met: $\mathrm{TJC} \leq 1 ; \mathrm{SJC} \leq 1$; enthesitis count $\leq 1$; PASI $\leq 1$; PtGA $\leq 20 \mathrm{~mm}$; patient pain (Pain) $\leq 15 \mathrm{~mm}$; and HAQ $\leq 0.5$.

2. DAPSA remission where DAPSA $\leq 4: \mathrm{TJC}+\mathrm{SJC}+\mathrm{PtGA}(\mathrm{cm})+$ Pain $(\mathrm{cm})+\mathrm{CRP}$ $(\mathrm{mg} / \mathrm{L})$.

3. cDAPSA remission where cDAPSA $\leq 4$ : TJC $+\mathrm{SJC}+\mathrm{PtGA}(\mathrm{cm})+$ Pain $(\mathrm{cm})$.

For LDA, six potential definitions were used:

1. MDA where any five of the seven cut points were required to be met: : $\mathrm{TJC} \leq 1 ; \mathrm{SJC} \leq 1$; enthesitis count $\leq 1$; PASI $\leq 1$; PtGA $\leq 20 \mathrm{~mm}$; Pain $\leq 15 \mathrm{~mm}$; and HAQ $\leq 0.5$. 
New targets for psoriatic arthritis

2. MDA joints where both the TJC and SJC cut points were required to be met with any $3 / 5$ of the remaining cut points (enthesitis, skin, PtGA, Pain and HAQ).

3. MDA skin where PASI $\leq 1$ was required plus $4 / 6$ remaining cut points (TJC, SJC, enthesitis, PtGA, Pain and HAQ).

4. MDA joints \& skin where the TJC, SJC and skin cut points were required to be met with any $2 / 4$ of the remaining cut points (enthesitis, PtGA, Pain and HAQ).

5. DAPSA low disease where DAPSA $\leq 14:=\mathrm{TJC}+\mathrm{SJC}+\mathrm{PtGA}(\mathrm{cm})+$ Pain $(\mathrm{cm})+\mathrm{CRP}$ $(\mathrm{mg} / \mathrm{L})$.

6. cDAPSA low disease where $\mathrm{cDAPSA} \leq 13:=\mathrm{TJC}+\mathrm{SJC}+\mathrm{PtGA}(\mathrm{cm})+$ Pain $(\mathrm{cm})$.

Descriptive statistics were produced for TJC, SJC, enthesitis, dactylitis, PASI, CRP and HAQ scores among patients meeting each definition of remission and LDA. Comparison of disease parameters in patients meeting vs. not meeting each definition was conducted using the Fisher's Exact test or the non-parametric Mann Whitney U test as appropriate. 
New targets for psoriatic arthritis

\section{RESULTS}

Baseline characteristics are shown in Table 1 . The mean age was 52 years and $53 \%$ of the patients were female with a mean disease duration of 5.5 years. The patients had predominantly mild/moderate disease activity with a mean TJC28 and SJC28 of 7.3 and 5.3, respectively. The presence of dactylitis and enthesitis was recorded in 26.4 and $32.3 \%$ of patients, respectively. Skin involvement was marginal with a mean (SD) PASI score of 2.3 (4.5).

The proportions of patients achieving each type of remission or LDA state at baseline, 6 and 12 months are shown in Figure 1. In all case, there was an increase in the proportion of patients achieving target at 6 and 12 months compared to baseline. The proportion of patients achieving VLDA was lower than those achieving DAPSA or cDAPSA remission at all time points suggesting that the VLDA criteria is more stringent. Indeed, patients who did not achieve DAPSA nor cDAPSA never achieved VLDA. Conversely, $75 \%$ and $53.3 \%$ of patients in DAPSA and cDAPSA remission, respectively, also achieved VLDA $(p<0.001)$. For the population, as a whole, no significant association was seen for DAPSA or cDAPSA remission vs VLDA achievement $(p=0.999)$. This lack of significance was likely driven by the low numbers of patients that were in either remission state. In regard to LDA achievement, the proportion of patients achieving any of the MDA-derived LDA outcomes at 6 and 12 months was substantially lower than those achieving LDA using either DAPSA LDA criteria (33.8-49.4\% vs 68.4-77.7\%). Among the MDA criteria, the proportion of patients achieving LDA at 6 or 12 months was similar between groups (40.3-49.4\%) and slightly lower for the MDA Joint \& Skin criteria (33.8$37.4 \%$ ). The proportions of patients achieving MDA also achieving MDA Joints, MDA Skin, MDA Joints \& Skin and DAPSA LDA were 83.8\%, 86.9\%, 70.7\% and 98.7\%, respectively.

Descriptive statistics for TJC, SJC, enthesitis, dactylitis and skin are presented in Supplementary Material. Patients achieving any remission target showed a significant reduction in TJC $(\mathrm{p}<0.001)$, SJC $(\mathrm{p}<0.001)$, and enthesitis $(\mathrm{P}<0.032)$ scores compared to non-achievers. However, the mean TJC and SJC scores were higher in the DAPSA-based outcomes compared to the MDA-based ones, both in patients in remission (TJC: 0.23-0.29 vs 0.09; SJC: 0.31-0.37 vs 0.16) and in LDA (TJC: $0.79-0.88$ vs $0.27-0.60$; SJC: $0.67-0.68$ vs $0.22-0.45$ ). There was residual dactylitis remaining in patients in VLDA (mean count: 0.5), DAPSA remission (mean count: 0.71), and cDAPSA (mean count: 0.58) remission despite inclusion of joint counts within all these measures. There was also some residual skin disease in patients in DAPSA (mean PASI: 0.43) and cDAPSA remission (mean PASI: 0.7). Among the 9 patients who were in DAPSA remission but not in VLDA, two had TJC $>1$, one had Pain $>15 \mathrm{~mm}$, two had a HAQ $>0.5$ and six had a PASI $>1$. Patients achieving any LDA target had significantly lower SJC, TJC, enthesitis, dactylitis and PASI scores compared to non-achievers $(\mathrm{p}<0.05)$ except for DAPSA LDA which had some residual skin disease $(\mathrm{p}=0.054)$.

CRP levels were numerically lower in patients in remission but did not reach statistical significance (not shown). We also investigated the impact of any residual activity on function 
New targets for psoriatic arthritis

using the HAQ score as a surrogate. Among patients with dactylitis at baseline, patients in remission with residual dactylitis had higher HAQ scores compared to patients with no remaining dactylitis. Indeed, mean (SD) HAQ scores were $0.31(0.09)$ vs. $0.07(0.15)(\mathrm{p}=0.029)$ for patients in VLDA, $0.50(0.18)$ vs $0.05(0.13)(\mathrm{p}=0.002)$ for patients in DAPSA remission and $0.59(0.30)$ vs $0.18(0.34)$ ( $\mathrm{p}=0.001)$ for patients in cDAPSA remission. HAQ scores were also numerically larger in patients in remission or in LDA with active skin disease (defined as PASI $\geq 12$ or $\mathrm{BSA} \geq 10 \%$ ) compared to patients without active skin disease, or in patients with enthesitis activity compared to patients without residual activity but they did not reach statistical significance (not shown).

\section{DISCUSSION}

The analysis of different remission and LDA targets in this real world clinical cohort shows that treating PsA patients with an efficacious therapy, in this case the anti-TNF agent golimumab, substantially increase the proportion of patients achieving a given target over a one-year period. Indeed, MDA is usually achieved in 39-61\% of PsA patients treated with an anti-TNF agent [1316]. Comparison of the several candidate measures of remission or LDA demonstrates that VLDA, or any of the MDA-derived LDA endpoints, are achieved by the lowest proportion of patients in this cohort in comparison to the DAPSA endpoints. This difference is best explained by the fact that DAPSA only focuses on peripheral joint disease (and CRP) while MDA covers additional extra-articular manifestations such as skin and enthesitis. Indeed, many patients in DAPSA or cDAPSA remission had residual skin activity indicating that joints and skin do not always resolve to the same extent. Although this residual skin activity was previously shown to have a negative impact of the quality of life in some patients [7], QoL data was not collected in this registry and we were unable to make this conclusion with respect to function, a proxy of quality of life, although a numerical but non-significant increase in HAQ was noted in those patients. We could demonstrate that residual dactylitis was associated with an increased HAQ score in patients in remission although dactylitis is not an index measure in any of the outcomes evaluated in this study even though it could be picked up as three swollen joints by some rheumatologists.

We used three different types of measures/constructs validated for psoriatic arthritis, the MDA and the adjusted versions (MDA skin, MDA joints and MDA joints \& skin as well as VLDA), DAPSA and cDAPSA. The MDA and adjusted MDA measures all use a modular approach where an individual cut-off for each domain is specified and depending on the measure used, several cut-offs need to be met. In contrast, the DAPSA measures sum the scores of the individual components into one final number. In DAPSA remission and LDA, higher levels of residual musculoskeletal disease were seen in comparison with the VLDA and the MDA 
New targets for psoriatic arthritis

measures. An active domain can be hidden when other domains are relatively unaffected, resulting in the inclusion of patients with active disease within the group of patients seen as in remission or low disease activity state.

Not all measures used in this comparison included an inflammatory marker. DAPSA includes CRP which cDAPSA and MDA measures do not. Our data suggest that the inclusion of CRP may not be necessary as we were unable to detect any impact of CRP on the achievement of remission or LDA or on HAQ scores. A target without an inflammatory marker would be more practical in clinical practice and in cases where routine laboratory assessment is not needed for other reasons. One limitation of this study is that this registry only measure disease activity in 28 joints while a full 66/68 joints assessment would normally be required [8]. It should be noted that, when PsA was included in the BioTRAC registry in 2005, a complete joint assessment was not considered as standard of care in the majority of Canadian community centers compared to tertiary care centers [17]. Studies have shown that the two are highly correlated and that differences between the simplified joint counts and the full 66/68 assessment are rather small in terms of sensitivity and specificity, at least in patients with polyarticular PsA [18]. One possible consequence would be an overestimation of the percent of patients achieving these outcomes. One way to estimate this impact would be to look at baseline values there must be clinical symptoms that would warrant the initiation of biologic therapy even if those patients were theoretically in remission or LDA. Indeed, we found that 3-5\% of patients were in remission at baseline while 5.6-23.6\% were in LDA. Moreover, a larger proportion of patients were in remission/LDA at baseline using the DAPSA-based scores suggesting that the latter could be more sensitive to the 28 joints scoring assessment than the MDA-derived scores.

In conclusion, this study shows that using an MDA-based outcome that covers more manifestations of PsA is stricter than only assessing peripheral arthritis. It remains to be determined whether meeting a strict target such as VLDA will lead to better long-term outcomes, such as radiographic progression, quality of life and function, in comparison with less stringent targets. 
New targets for psoriatic arthritis

Table 1. Baseline characteristics

\begin{tabular}{|c|c|}
\hline Baseline Parameters & $\begin{array}{c}\text { Total } \\
(\mathrm{N}=201)\end{array}$ \\
\hline \multicolumn{2}{|l|}{ Socio-demographics } \\
\hline \multicolumn{2}{|l|}{ Gender $^{\mathrm{a}}, \mathrm{n}(\%)$} \\
\hline Male & $88(46.8 \%)$ \\
\hline Female & $100(53.2 \%)$ \\
\hline Age (years), mean (SD) & $52.0(13.0)$ \\
\hline \multicolumn{2}{|l|}{ Disease Parameters } \\
\hline Disease duration (years), mean (SD) & $5.5(6.9)$ \\
\hline DAS28-ESR, mean (SD) & $4.3(1.5)$ \\
\hline DAS28-CRP, mean (SD) & $4.3(1.3)$ \\
\hline TJC28, mean (SD) & $7.3(6.7)$ \\
\hline SJC28, mean (SD) & $5.3(4.2)$ \\
\hline MDGA (VAS cm), mean (SD) & $5.2(2.1)$ \\
\hline PtGA (VAS mm), mean (SD) & $53.1(25.4)$ \\
\hline AM stiffness ${ }^{\mathrm{b}}(\mathrm{min})$, mean (SD) & $37.1(41.1)$ \\
\hline HAQ, mean (SD) & $1.0(0.7)$ \\
\hline Pain (VAS mm), mean (SD) & $52.0(25.2)$ \\
\hline PASI, mean (SD) & $2.3(4.5)$ \\
\hline Presence of enthesitis, n (\%) & $65(32.3 \%)$ \\
\hline Enthesitis count ${ }^{\mathrm{c}}$, mean (SD) & $5.1(3.9)$ \\
\hline Presence of dactylitis, n (\%) & $53(26.4 \%)$ \\
\hline Dactylitis count ${ }^{\mathrm{d}}$, mean (SD) & $4.7(4.4)$ \\
\hline $\mathrm{ESR}(\mathrm{mm} / \mathrm{h})$, mean $(\mathrm{SD})$ & $17.6(16.7)$ \\
\hline CRP (mg/L), mean (SD) & $12.2(27.0)$ \\
\hline \multicolumn{2}{|l|}{ Medications, n (\%) } \\
\hline Previous biologic & $13(6.5 \%)$ \\
\hline Previous DMARD & $183(91.0 \%)$ \\
\hline Previous corticosteroid & $39(19.4 \%)$ \\
\hline Concomitant DMARD & $156(77.6 \%)$ \\
\hline Concomitant Methotrexate & $125(62.2 \%)$ \\
\hline Concomitant corticosteroid use & $13(6.5 \%)$ \\
\hline
\end{tabular}

${ }^{\text {a }}$ Percentage based on available data

${ }^{\mathrm{b}}$ Capped at 120 minutes.

${ }^{\mathrm{c}}$ Among patients with enthesitis.

${ }^{\mathrm{d}}$ Among patients with dactylitis.

CRP, C-reactive protein; DAS28, Disease Activity Score; DMARD, disease-modifying anti-rheumatic drug; ESR, erythrocyte sedimentation rate; HAQ, Health assessment questionnaire; MDGA, Physician Global Assessment of Disease Activity; PASI, Psoriasis Area and Severity Index; PtGA, Patient Global Assessment of Disease Activity; SD, standard deviation; SJC, Swollen joint count; TJC, Tender joint count; VAS, Visual Analog Scale. 
New targets for psoriatic arthritis

Figure 1: Achievement of in remission and LDA at baseline and after 6 or 12 months of golimumab therapy

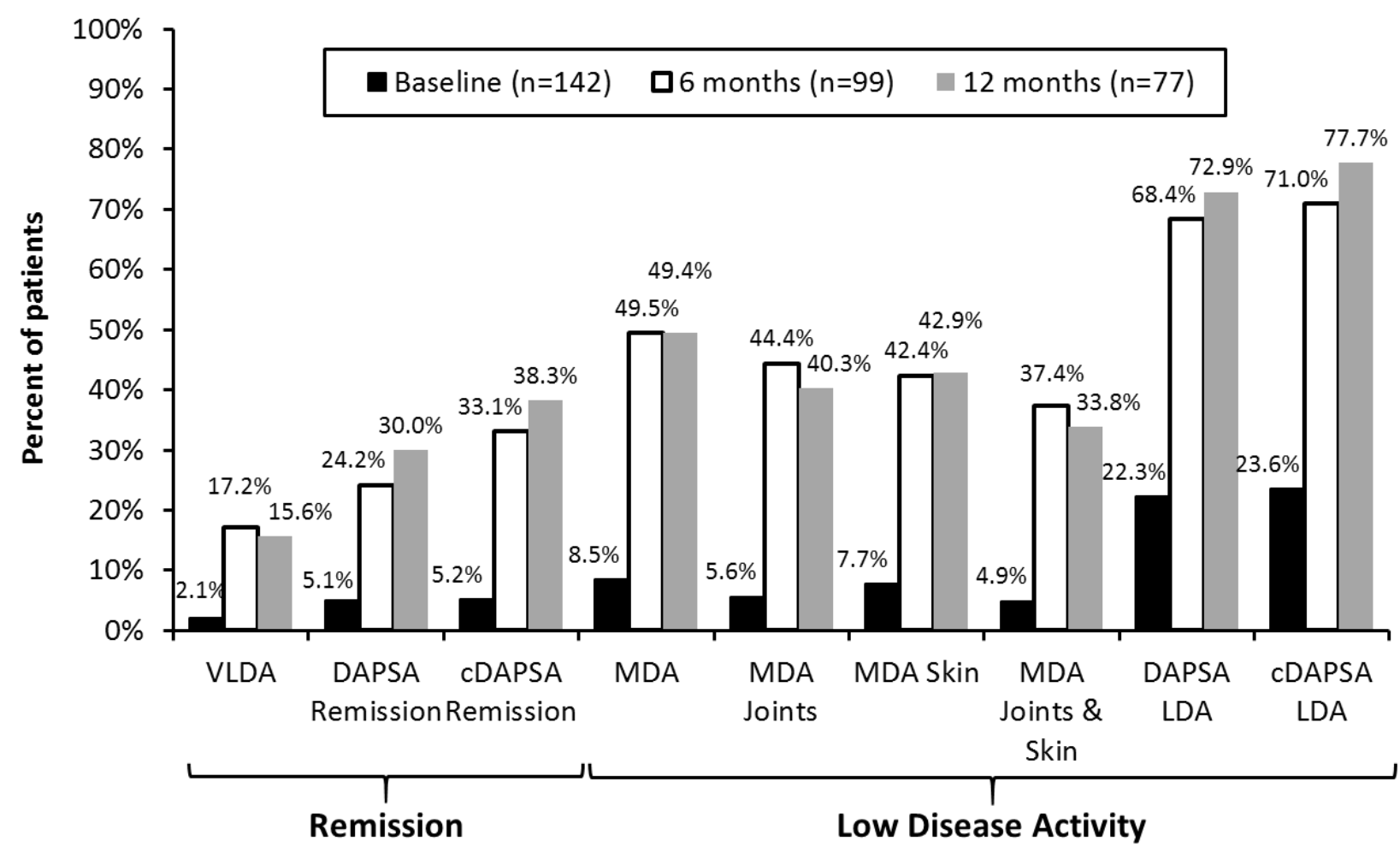


Supplementary Material: Descriptive Statistics for TJC, SJC, enthesitis count, dactylitis count and PASI score by outcome. a. VLDA; b. DAPSA Remission; c. cDAPSA Remission; d. DAPSA LDA; e. cDAPSA LDA; f. MDA 5/7; g. MDA Joint; h. MDA Skin; i. MDA Joint and Skin. Data is mean, error bars are S.E. and box represents the 95\% C.I.
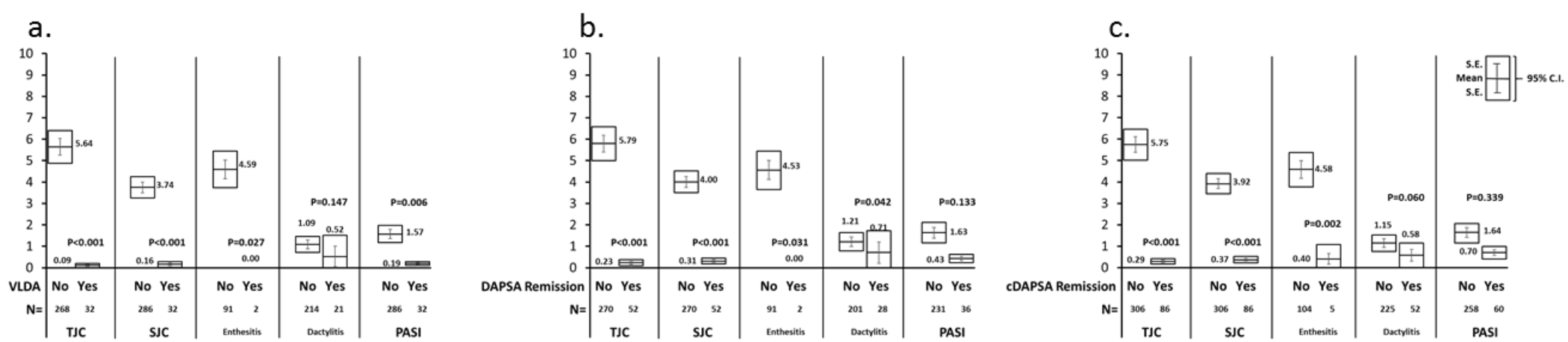

$$
\text { d. }
$$

e.
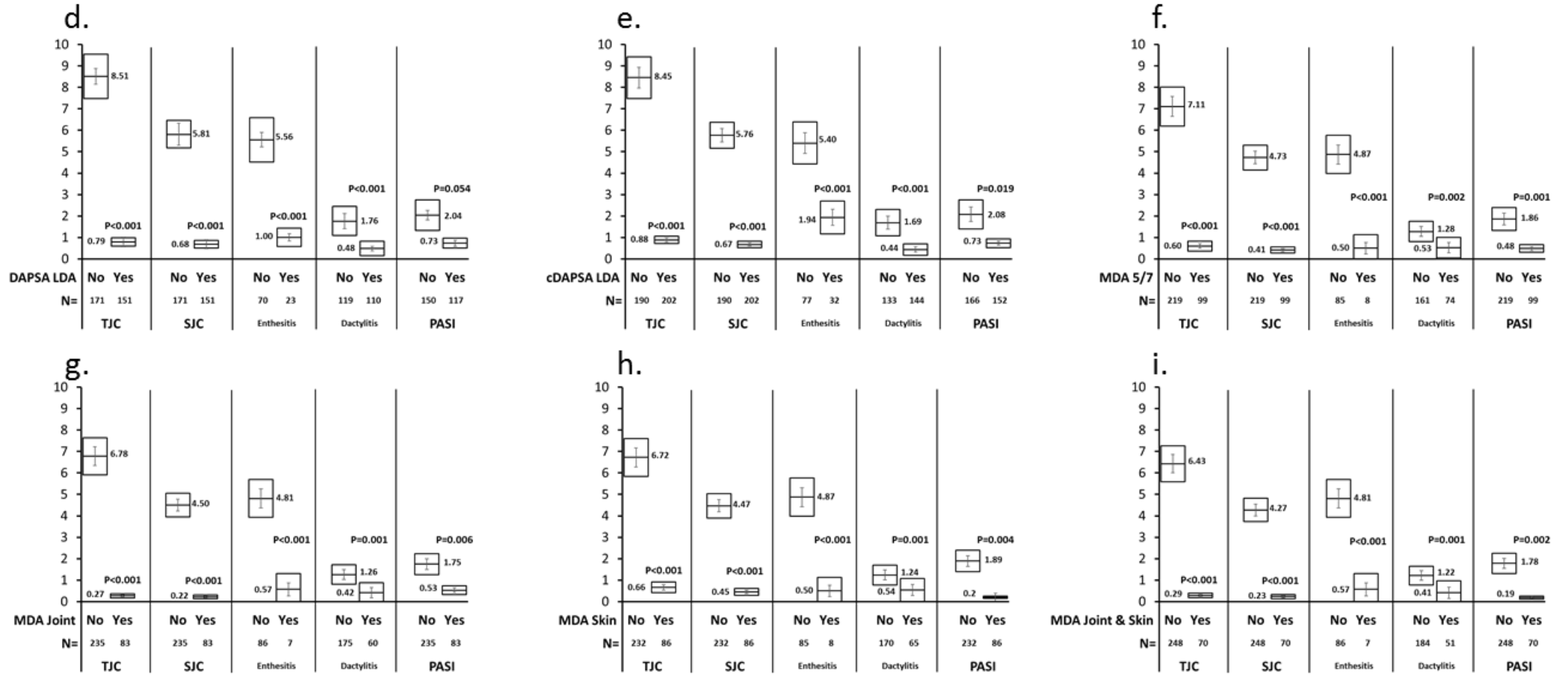
New targets for psoriatic arthritis

\section{REFRENCES}

1. Coates, L.C., R. Murphy, and P.S. Helliwell, New GRAPPA recommendations for the management of psoriasis and psoriatic arthritis: process, challenges and implementation. Br J Dermatol, 2016. 174(6): p. 1174-8.

2. Gossec, L., et al., Management of psoriatic arthritis in 2016: a comparison of EULAR and GRAPPA recommendations. Nat Rev Rheumatol, 2016. 12(12): p. 743-750.

3. Coates, L.C. and P.S. Helliwell, Defining Low Disease Activity States in Psoriatic Arthritis using Novel Composite Disease Instruments. J Rheumatol, 2016. 43(2): p. 371-5.

4. Coates, L.C., et al., Remission in psoriatic arthritis-where are we now? Rheumatology (Oxford), 2017.

5. Coates, L.C., et al., New GRAPPA and EULAR recommendations for the management of psoriatic arthritis. Rheumatology (Oxford), 2017. 56(8): p. 1251-1253.

6. Smolen, J.S., et al., Treating axial spondyloarthritis and peripheral spondyloarthritis, especially psoriatic arthritis, to target: 2017 update of recommendations by an international task force. Ann Rheum Dis, 2017.

7. van Mens, L.J.J., et al., Ideal target for psoriatic arthritis? Comparison of remission and low disease activity states in a real-life cohort. Ann Rheum Dis, 2017.

8. Coates, L.C., J. Fransen, and P.S. Helliwell, Defining minimal disease activity in psoriatic arthritis: a proposed objective target for treatment. Ann Rheum Dis, 2010. 69(1): p. 48-53.

9. Smolen, J.S., M. Schoels, and D. Aletaha, Disease activity and response assessment in psoriatic arthritis using the Disease Activity index for PSoriatic Arthritis (DAPSA). A brief review. Clin Exp Rheumatol, 2015. 33(5 Suppl 93): p. S48-50.

10. Coates, L.C., et al., The dynamics of response as measured by multiple composite outcome tools in the TIght COntrol of inflammation in early Psoriatic Arthritis (TICOPA) trial. Ann Rheum Dis, 2017. 76(10): p. 1688-1692.

11. Schoels, M.M., et al., Disease activity in psoriatic arthritis (PSA): defining remission and treatment success using the DAPSA score. Ann Rheum Dis, 2016. 75(5): p. 811-8.

12. Thorne, C., et al., Effectiveness and safety of infliximab in rheumatoid arthritis: analysis from a Canadian multicenter prospective observational registry. Arthritis Care Res (Hoboken), 2014. 66(8): p. 1142-51.

13. Rahman, P., et al., Real-world validation of the minimal disease activity index in psoriatic arthritis: an analysis from a prospective, observational, biological treatment registry. BMJ Open, 2017. 7(8): p. e016619.

14. Kavanaugh, A., et al., Radiographic Progression of Patients With Psoriatic Arthritis Who Achieve Minimal Disease Activity in Response to Golimumab Therapy: Results Through 5 Years of a Randomized, Placebo-Controlled Study. Arthritis Care Res (Hoboken), 2016. 68(2): p. 267-74.

15. Mease, P.J., et al., Application and modifications of minimal disease activity measures for patients with psoriatic arthritis treated with adalimumab: subanalyses of ADEPT. J Rheumatol, 2013. 40(5): p. 647-52.

16. Perrotta, F.M., A. Marchesoni, and E. Lubrano, Minimal Disease Activity and Remission in Psoriatic Arthritis Patients Treated with Anti-TNF-alpha Drugs. J Rheumatol, 2016. 43(2): p. 3505.

17. Gladman, D.D., C. Ritchlin, and P.S. Helliwell, Psoriatic arthritis clinical registries and genomics. Ann Rheum Dis, 2005. 64 Suppl 2: p. ii103-5.

18. Englbrecht, M., et al., Measuring joint involvement in polyarticular psoriatic arthritis: an introduction of alternatives. Arthritis Care Res (Hoboken), 2010. 62(7): p. 977-83. 
New targets for psoriatic arthritis 